\title{
Advanced Approach for Animal Recognition System Based On Convolution Neural Network
}

\author{
Chinmay S. Borkar ${ }^{1}$, Rupali Dasarwar ${ }^{2}$, Pratibha Motwani $^{3}$ \\ ${ }^{1} P G$ Student, ${ }^{2,3}$ Asst. professor \\ Wainganga college of engineering and management, Nagpur, India, 440012.
}

DOI: 10.46335/IJIES.2020.5.10.2

\begin{abstract}
In this paper, the animal images are processed using convolution neural network (CNN).This method is compared with animal recognition methods like Linear discriminate analysis (LDA),Principal compone -nt analysis(PCA), Support vector machine(SVM),Local binary pattern histogram (LBPH). For many years in order to study the eco-system of the endangered species we were relaying only on our physical presence. There was huge risk of life and on top of that due to the lack of the automated systems, overall data gathered was not sufficient to conduct the thorough study on the endangered species.For experimental purpose we are creating database of the animals in which there are separate class for each and every animal has 100 images.
\end{abstract}

Keywords-convolution neural network(CNN),SVM,LDA Local binary pattern histogram $(L B P H)$.

\section{I- INTRODUCTION}

Wildlife traditionally refers to undomesticated animal species, but has come to include all organisms that grow or live wild in an area without being introduced by humans. Wildlife can be found in all ecosystems. Deserts, forests, rain forests, plains, grasslands and other areas including the most developed urban areas, all have distinct forms of wildlife. While the term in popular culture usually refers to animals that are untouched by human factors, most scientists agree that much wildlife is affected by human activities. Humans have historically tended to separate civilization from wildlife in a number of ways including the legal, social, and moral sense. Some animals, however, have adapted to suburban environments.

This includes such animals as domesticated cats, dogs, mice, and gerbils. Some religions declare certain animals to be sacred, and in modern times concern for the natural environment has provoked activists to protest against the exploitation of wildlife for human benefit or entertainment. The global wildlife population decreased by 52 percent between 1970 and 2014. According to a report by the World Wildlife Fund.

Wild Animal Monitoring Challenges -There are a few notable challenges in the wild animal detecting monitoring.

Data transferring-There won't be internet in the wild. We have cellular or radio. But we cannot afford sending/receiving too much data through cellular/radio channels.

AI model on a Raspberry Pi Zero -There are sophisticated AI running in a cloud. There are powerful AI models running on a desktop machine. But AI model running on a Raspberry Pi or even a RPi Zero.

Hardware reliability in the wild -Yes, wild could be really wild. The tiger is the largest cat species, most recognizable for its pattern of dark vertical stripes on reddish-orange fur with a lighter underside. The species is classified in the genus Panther with the lion, jaguar, leopard, and snow leopard. It is an apex predator, primarily praying on ungulates such as deer and bovid. 
Neural Network in Computer-Artificial neural networks (ANN) or connectionist systems are computing systems inspired by the biological neural networks that constitute animal brains. The neural network itself is not an algorithm, but rather a framework for many different machine learning algorithms to work together and process complex data inputs. Such systems "learn" to perform tasks by considering examples, generally without being programmed with any task-specific rules. Because of their ability to reproduce and model nonlinear processes, artificial.Neural Networks have found many applications in a wide range of disciplines. Application areas include system identification and control. While verification and identification often share the same classification algorithms, both modes target distinct applications. In order to better understand the animal detection and recognition task and its difficulties, the following factors must be taken into account, because they can cause serious performance degradation in animal detection and recognition systems.

\section{II- METHODOLOGY}

In order to find the efficient solution on the abovementioned problem we have developed a system which will take footage from CCTV camera on which the image processing will take place the data obtained from the image processing could then be used to identify the name of the animal and their species location. To do that we would be using same camera to detect the multiple animals. The project is based on raspberry pi and to do the image processing we have used machine leaning. The benefit of using machine learning is that whenever the image is being processed, the systems train itself repeatedly which would lead tithe creation of the database. So, if in near future an image matches with the database the accuracy of the output will be more. To do this we have to compare the current image (to be processed) with the master image. The master images will contain all those images that we have used to train our system. All these images, they comprise the data set and the technique which is used to retrieve this dataset is called Neural Network. The basic working principle of neural network is that whenever the system comes across the new image, it checks this new image with all the folders of the datasets and checks whether which images of the dataset matches more exactly to the uploaded new image, in our case the system Will be trained with the photos of the desired animals. The neural network will then be trained multiple times until it gives us the desired output.

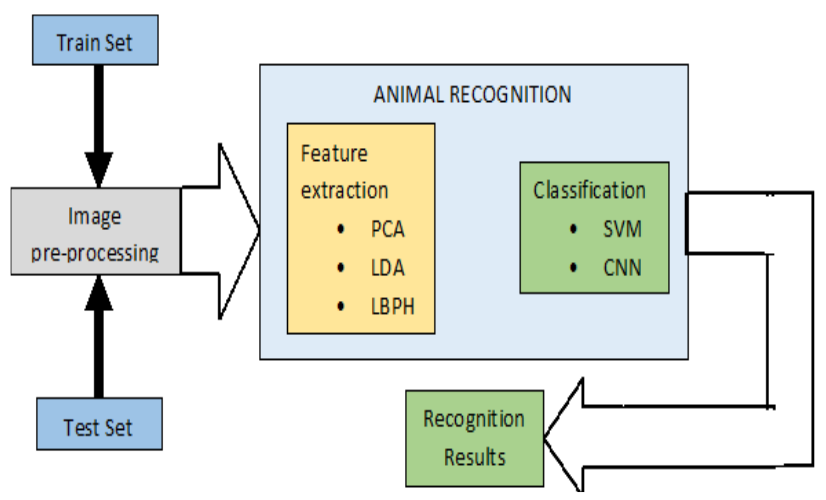

Fig 1:- Working diagram

Once the desired output is obtained then we could use our system for the real time usage with the help of the obtained dataset. The system then will compare the new image with this dataset and will check which characteristics of the uploaded image matches more with that of dataset and then after the comparison the image will be saved in the folder of that animal whose image matches more with the uploaded image. The comparison which the system does is basically the unique characteristic of machine learning. In machine learning we have used viola jones algorithm to detect face along with that we have used the feature extraction. Feature extraction will identify the characteristics of the animals which are unique to them. Example:- To detect tiger, the system will be trained to recognize the unique pattern of the stripes of tigers, this identification will be done by feature extraction algorithm now this recognized image of tiger will be retrieved by the neural network and will save the image of tiger in it's specified folder of dataset so that the next time the system comes across the image of tiger it would easily detect that the image is of tiger more efficiently thus reducing the errors.

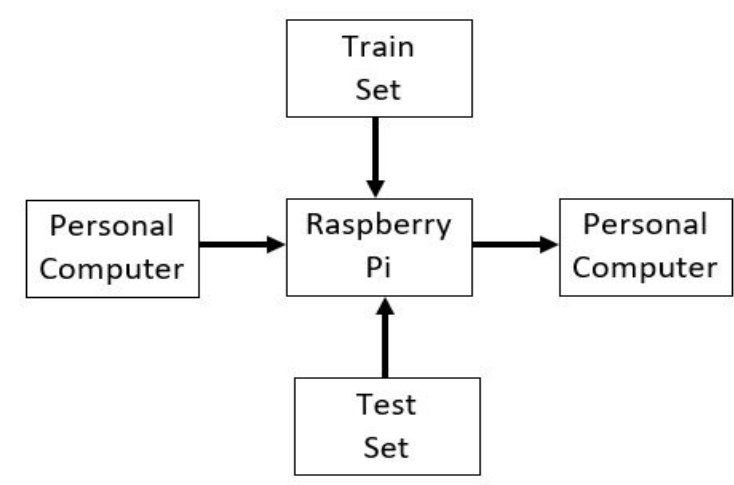

Fig 2 - Block Diagram of System.

In the proposed system, the image recognition algorithm (image classier) takes the image (or a patch of the image) 
as input and outputs what the image contains. In other words, the output is a class label (fox, tiger, blue bull, etc.)

\section{III- DESIGN}

The Convolutional Neural Network is similar in architecture to the original LeNet (Convolutional Neural Network in Python) and classifies an input image into categories: fox, wolf, bear, hog or deer (the original LeNet was used mainly for character Recognition tasks) As it is evident from the figure above with a fox image as input, the network correctly assigns the probability for fox among all five categories.

There are four main operations in the $\mathrm{CNN}$ :

- Convolution.

- Non Linearity (ReLU).

- Pooling or Sub-Sampling.

- Classification (Fully Connected Layer).

These operations are the basic building blocks of every Convolutional Neural Network.

Because of their ability to reproduce and model nonlinear processes, artificial.Neural Networks have found many applications in a wide range of disciplines. Application areas include system identification and control. Process information in a way that is inspired by the way biological nervous systems, such as the brain, process information. The key element of this paradigm is the novel structure of the information processing system. Artificial Neural Networks have been used to accelerate reliability analysis of infrastructures subject to natural disasters and to predict foundation settlements.ArtificialNeural Networks have also been used for building black-box models in geosciences: hydrology, [ocean modelling and coastal engineering, and geomorphology.

\section{IV-CONCLUSION}

This paper provides a overview of the comparison between the well-known methods of animal recognition system. The given data is used to create a set in the database. The performance is obtained by the help of different test images. The experimental result shows that the LBPH algorithm provides better results than PCA, LDA and SVM for large training set. On the other hand, SVM is better than PCA and LDA for small training data set. The best experimental results of animal recognition were obtained using the proposed CNN. The obtained experimental results of the performed experiments show that the proposed $\mathrm{CNN}$ gives the best recognition rate for a greater number of input training images (accuracy of about $98 \%$ ). When the image is divided into more windows the classification results should be better. On the other hand, the computation complexity will increase.

In the future work, we plan to perform experiments and also tests of more complex algorithms with aim to compare the presented approaches (PCA, LDA, SVM and LBPH) with other existing algorithms (deep learning). We are also planning to investigate reliability of the presented methods by involving larger databases of animal images.

\section{V- ACKNOWLEDGMENT}

This publication is a result of acknowledge the sincere efforts and valuable time given by the professors of electronics branch of Wainganga college of engineering and management, who provided insight and expertise that greatly assisted the research. I would like to express my special thanks of gratitude to my teacher as well as our principal who also helped me to do this wonderful project, which also help me in doing lot of research and I came to know about so many new things. I am really thankful to them.

\section{REFERENCES}

[1] HayderYousif, Jianhe Yuan, Roland Kays, Zhihai He, "Fast Human- Animal Detection from Highly Cluttered Camera-Trap Images UsingJoint Background Modeling and Deep Learning Classi_cation", IEEE2017.

[2] Manohar N., Subrahmanya S., Bharathi R. K., Sharath Kumar Y. H.,Hemantha Kumar G., Vecognition and Classi_cation of Animals basedon Texture Features through Parallel Computing", IEEE Second International Conference on Cognitive Computing and Information Processing (CCIP) 2016.

[3] E. Surendar, Vimin M. Thomas, \Animal Tracking Using Background Subtraction on Multi Threshold Segmentation", IEEE International Con-ference on Circuit, Power and Computing Technologies [ICCPCT] 2016.

[4] Guobin Chen, Tony X. Han, Zhihai He, Roland Kays, and Tavis For-rester, "Deep Convolution Neural Network Based Species Recognition ForWild Animal Monitoring", 978-1-4799-5751-4/14/ 31.00 dollars c 2014IEEE, ICIP 2014.

[5] David Forslund and Jon Bjarkefur, Wight Vision Animal Detection",2014 IEEE Intelligent Vehicles Symposium (IV) June 8-11, 2014.Dear-born, Michigan, USA. 